\title{
Historie und Organisation des Monitorings
}

Das Monitoring ist eine eigenständige Aufgabe in der amtlichen Überwachung gemäß §§50-52 LFGB [2]. Die im Zeitraum 2011 bis 2015 vorgesehenen Untersuchungen sind in der AVV Monitoring 2011-2015 [1] festgelegt. Das Monitoring von Lebensmitteln wird in dieser Form bereits seit 1995 durchgeführt. Von 1995 bis 2002 wurden die Lebensmittel auf der Basis eines aus dem Ernährungsverhalten der Bevölkerung entwickelten Warenkorbs [3] ausgewählt.

Seit 2003 wird das Lebensmittel-Monitoring zweigeteilt durchgeführt. Um die Situation hinsichtlich der Rückstände und der Kontamination unter repräsentativen Beprobungsbedingungen weiter verfolgen zu können, werden die Lebensmittel entsprechend den Vorgaben der jeweils geltenden Allgemeinen Verwaltungsvorschrift zur Durchführung des Monitorings weiterhin aus dem repräsentativen Warenkorb der Bevölkerung ausgewählt (Warenkorb-Monitoring). Ergänzend dazu werden spezielle Themenbereiche in Form von Projekten bearbeitet (Projekt-Monitoring), um zielorientiert aktuelle Fragestellungen zu untersuchen und Kenntnislücken für die Risikobewertung zu schließen.

Seit 2009 werden im Warenkorb-Monitoring auch die Vorgaben eines speziell zur Untersuchung auf Rückstände von Pflanzenschutzmitteln konzipierten nationalen
Monitorings [4] berücksichtigt. Weiterhin wird jährlich das mehrjährige koordinierte Kontrollprogramm (KKP) der EU zu Pestizidrückständen in oder auf Lebensmitteln $[5,6,7]$ in das Warenkorb-Monitoring integriert.

Bei der Festlegung der im Warenkorb-Monitoring zu untersuchenden Stoffe wurden darüber hinaus Erkenntnisse über die Kontaminations- bzw. Rückstandssituation sowie Empfehlungen aus früheren Untersuchungen für eine erneute Überprüfung des Vorkommens dieser Stoffe berücksichtigt.

Auf der rechtlichen Grundlage der §§ 50-52 LFGB [2] wurden beginnend mit dem Jahr 2010 neben Lebensmitteln auch kosmetische Mittel und Bedarfsgegenstände im Rahmen des Warenkorb-Monitorings untersucht.

Die ausgewählten Erzeugnisse werden durch die amtlichen Untersuchungseinrichtungen der Länder analysiert. Die Organisation des Monitorings, die Erfassung und Speicherung der Daten, die Auswertung der Monitoring-Ergebnisse sowie deren jährliche Berichterstattung obliegen dem Bundesamt für Verbraucherschutz und Lebensmittelsicherheit (BVL).

Eine Übersicht der seit 1995 untersuchten Lebensmittel, kosmetischen Mittel und Bedarfsgegenstände ist im Internet unter http://www.bvl.bund.de/monitoring verfügbar. 